\title{
Erratum to: Aesthetic Surgery of the Breast
}

\author{
Toma T. Mugea, Melvin A. Shiffman
}

\section{Erratum to}

T.T. Mugea, M.A. Shiffman (eds.), Aesthetic Surgery of the Breast, DOI 10.1007/978-3-662-43407-9

The link for the videos provided in the FM pages is not working.

The FM pages were updated with present link (http://extras.springer.com) and included "Extras Online" logo in the cover and uploaded the video files of the extra materials server. 